\title{
Notas sobre la política, el Estado, la DEMOCRaCia y EL SUJETO. Tensionando ALGUNAS CATEGORÍAS DE JACQUES RANCIÈRE PARA PENSAR LA ÚLTIMA DÉCADA ARGENTINA
}

\section{Fernando Daniel Chávez Solca*}

RESUMEN. El presente trabajo se propone esbozar algunas notas a partir del filósofo Jacques Rancière para pensar la política, el Estado, la democracia y el sujeto. El tratamiento de cada una de estas cuestiones a la luz de la experiencia argentina de los últimos años nos posibilitará desentrañar la potencialidad analítica de dichas categorías y al mismo tiempo tensionarlas para mostrar sus límites y la necesidad de reformularlas, lo cual habilitaría una forma diferente de pensar el kirchnerismo.

Palabras clave. Rancière, kirchnerismo, política, Estado, democracia, sujeto.

\section{Notes on Politics, State, Democracy and Subject. Questioning CATEGories of JaCQues RAnCIËre} TO THINK THE LAST ARGENTINIAN DECADE

Abstract. This paper aims to outline some notes about the thought of philosopher Jacques Rancière about politics, state, democracy and the subject. The treatment of each of these issues in light of argentinian experience of recent years enable us to disentangle the analytical potential of these categories. At the same time, we will tension its boundaries and the need to reformulate them, thus enabling a different way of thinking the kirchnerism.

* Docente en la Universidad de Villa María e investigador del Consejo Nacional de Investigaciones Científicas y Técnicas (Conicet). Correo electrónico: ferchavezsolca@ gmail.com 
KEY WORDS. Rancière, kirchnerism, politics, state, democracy, subject.

\section{INTRODUCCIÓN}

El presente artículo tiene por propósito reflexionar a partir de la obra del pensador francés Jacques Rancière. Particularmente nos interesa recuperar de manera crítica los aportes realizados desde una mirada posestructuralista con dos propósitos, uno de carácter teórico y otro de carácter empírico-analítico. El primero busca presentar y repensar sus categorías y constructos teóricos (como el presupuesto de la igualdad, la división entre política y policía y la emergencia del sujeto como proceso de desclasificación o desidentificación), discutiendo el modo en que algunos de ellos han sido interpretados mayoritariamente. El segundo propósito se relaciona con "utilizar" las categorías mencionadas para analizar y pensar dos grandes ejes: la democracia y el Estado en el marco del proceso que atraviesa Argentina (y América Latina) en la última década.

Creemos que la teoría política posfundacional en general y los conceptos producidos por Rancière en particular pueden ayudarnos a echar luz sobre lo acontecido en los últimos años en Argentina, alejándonos de las miradas tradicionales, a menudo normativas, que sitúan las experiencias recientes en el lugar del error, la desviación y la anomalía. En este sentido, una gramática que nos habilite a reconocer la especificidad o particularidad del proceso actual sin intentar encausarlo en la vía de los países del cuadrante noroccidental, que reconozca la dimensión conflictual de la política, puede ser de provecho en el desarrollo de una comprensión diferente de este proceso. Simultáneamente, entendemos que el análisis de la experiencia argentina pondrá en evidencia algunos límites de la propuesta rancieriana, lo que nos obligará a tensionar sus postulados y reformularlos para intentar pensar lo acontecido.

A partir de lo dicho, el trabajo se estructurará con base en cuatro puntos. Primero, partiendo de la distinción que hace Rancière entre política y policía, intentaremos mostrar la co-constitutividad de ambos polos y los efectos que tiene ello para pensar la situación argentina. 
Segundo, desarrollaremos una breve reflexión sobre el exiguo lugar que el Estado ocupa en el pensamiento rancieriano y buscaremos establecer una salida para dicha limitación. Tercero, dedicaremos algunas líneas a pensar la cuestión de la democracia desde las ideas de Rancière y las habilitaciones que ese modo de abordar la cuestión nos permite conjugar en la actual coyuntura. Cuarto y último, repasaremos la noción de sujeto que Rancière elabora en varios pasajes de su obra, proponiendo un ligero desplazamiento, en consonancia con lo sostenido en los puntos anteriores, que nos permita entender al sujeto no como un emergente fugaz, momentáneo, que irrumpe para luego desvanecerse, sino como una figura permanente que interviene en la coyuntura de formas sutiles pero persistentes.

Uno de los aspectos más difundidos de la obra de Rancière es la distinción que este autor realiza entre dos lógicas o procesos: política y policía. La última refiere a una tecnología de gobierno, es el modo en que se cuentan las partes y es el proceso de distribución de lugares y establecimiento de un orden (Rancière, 2010a). En otras palabras, son todos y cada uno de los procedimientos de gobierno mediante los cuales se pretende ordenar la vida comunitaria, estableciendo jerarquías, funciones, papeles a los actores intervinientes. En sus propias palabras:

la policía es primeramente un orden de los cuerpos que define las divisiones entre los modos del hacer, los modos del ser y los modos del decir, que hace que tales cuerpos sean asignados por su nombre a tal lugar y a tal tarea; es un orden de lo visible y lo decible que hace que tal actividad sea visible y que tal otra no lo sea, que tal palabra sea entendida como perteneciente al discurso y tal otra al ruido (Rancière, 2010a: 44-45).

Por su lado, la política es justamente la actividad que rompe con dicha configuración de lo sensible, hace emerger a la esfera pública a aquellos que no tienen parte, vuelve palabra lo que hasta entonces era sólo 
ruido, obliga a hacer nuevamente la cuenta, es decir, la política es toda práctica que pone en marcha e intenta verificar el presupuesto de la igualdad, cuestionando toda división producida por el orden policial. Así, la política emerge para reparar un daño que toda ficción de desigualdad efectúa sobre aquellos participantes a los que no se les reconoce su lugar como pares; muestra el desacuerdo con toda distribución de lugares: evidencia la fragilidad y precariedad de cualquier forma de orden y expone que la cuenta siempre resulta ser errónea.

Para arribar a ello, el punto de partida de la política es el presupuesto de la igualdad desde el que se sitúa Rancière, al entender que la multiplicidad de relaciones desiguales que existe en toda sociedad está estructurada sobre una base igualitaria, ya que para que alguien "superior" ordene, necesita que su inferior, en primer lugar, entienda lo que se le está ordenando y, en segundo lugar, comprenda que ello es una orden y, en cuanto tal, debe cumplirla (Rancière, 2007b). Ese doble gesto de entendimiento mutuo demuestra la igualdad de las inteligencias de cualquiera con cualquiera, ${ }^{1}$ y en consecuencia no hay posibilidad de sostener un fundamento natural-último de la desigualdad en las relaciones sociales que se traduzca en un reparto desigual de lugares en la sociedad, más que mediante la generación de ficciones. Así, cuando una de las partes asume la capacidad de decirle al otro lo que debe hacer, esa asunción es arrogada, sin un sustento trascendental que lo habilite o permita fijarlo de una vez y para siempre. A partir de ello, ese principio de igualdad no deja de horadar una y otra vez toda configuración policial que pretende mostrarse como natural,

\footnotetext{
${ }^{1}$ La cuestión de la igualdad es un tema que atraviesa transversalmente toda la obra de Rancière, pero particularmente es en El maestro ignorante (2007b) donde este autor expone de manera más acaba su concepción sobre dicha problemática. A partir de la experiencia docente de Joseph Jacotot en los Países Bajos, Rancière nos señala la igualdad de las inteligencias que sólo se ve interrumpida por el principio de la explicación o del atontamiento que introduce una relación jerárquica y de subordinación entre el maestro explicador y el alumno ignorante en donde antes había igualdad, y que esa división resulta innecesaria e inconducente (Rancière, 2007b). Con ello señala que toda desigualdad es una ficción que pretende legitimarse para perdurar. Las implicancias políticas de este aspecto es lo que intentamos poner en juego en lo que estamos desarrollando.
} 
intentando determinar quiénes cuentan y quiénes no, quiénes tienen palabra y quiénes no, quiénes son visibles y quiénes no.

Entonces, la política muestra el radical desacuerdo por el modo en que se hace la cuenta, pero no como una mera disputa de intereses entre partes que se reconocen como tales y que a partir de la negociación racional pueden llegar a un acuerdo. El desacuerdo, por decirlo de algún modo, es anterior, es una disputa por las partes mismas que están legitimadas a sentarse en la mesa, por el reconocimiento igualitario, por el derecho a ser contadas como partes. En la misma línea, no es un desacuerdo por hacer una cosa u otra, entre quienes dicen blanco y quienes dicen negro, es una disputa por el sentido mismo de lo negro, un desacuerdo por el significado de los términos, una lucha por establecer lo común en sí mismo:

hay política porque quienes no tienen derecho a ser contados como seres parlantes se hacen contar entre éstos e instituyen una comunidad por el hecho de poner en común la distorsión, que no es otra cosa que el enfrentamiento mismo, la contradicción de dos mundos alojados en uno solo: el mundo en que son y aquel en que no son, el mundo donde hay algo "entre" ellos y quienes no los conocen como seres parlantes y contables, y el mundo donde no hay nada (Rancière, 2010a: 42).

Trasladándolo a la realidad contemporánea argentina, la disputa por la ley de servicios de comunicación audiovisual ${ }^{2}$ parece ofrecernos un

\footnotetext{
2 Nos referimos a la Ley 26.522 promulgada el 10 de octubre de 2009, conocida popularmente como Ley de Medios, que reemplazó la Ley de Radiodifusión 22.285, instituida en 1980 por la dictadura cívico-militar, la cual establece las normas que rigen el funcionamiento y la distribución de licencias de los medios radiales y televisivos en Argentina. Los puntos que particularmente generaron la resistencia de los grupos multimediáticos concentrados fueron el artículo 45, que impone una limitación sobre la propiedad de licencias para evitar la concentración de éstas en pocas manos, y el artículo 161, que dispone los plazos para que esos grupos se ajusten a la ley. Esos puntos, junto con la intransferibilidad de las licencias (art. 41), fueron suspendidos provisoriamente por las medidas cautelares presentadas por el Grupo Clarín a la justicia hasta que ésta declaró la constitucionalidad de dichas disposiciones y la total aplicabilidad de la ley, luego de muchos años de conflicto.
} 
buen caso para ser leído en estos términos. A partir de su sanción (y un poco antes también) en Argentina comenzó a discutirse el sentido de la "libertad de expresión". Simplificando el debate, podríamos sugerir que la forma asumida por esa disputa fue entre quienes entendían la libertad de expresión ligada a la libertad de empresa o, dicho más brutalmente, aquellos que sostenían que la libertad de expresión significaba tener todos los medios que se deseara tener sin ningún tipo de restricciones; y aquellos que entendían que la libertad de expresión significaba una mayor pluralidad de voces, que todos los actores interesados pudieran tener su espacio en la grilla televisiva y radial. Digámoslo de este modo: la disputa por el sentido mismo de la "libertad de expresión" permitió visibilizar, reconocer en pie de igualdad, a una multiplicidad de actores vinculados a los medios, como las pequeñas cooperativas, sectores de la población civil que reclaman su espacio y colectivos varios, que bajo el orden policial vigente no contaban, quienes no tenían derecho a hacerse contar como seres parlantes se hicieron contar; con lo cual se mostró la contradicción del ordenamiento policial. Es en ese sentido que la disputa por la ley de medios asume su carácter político, al darle parte a la parte que no tenía parte, con lo cual modificó lo dado.

Ahora bien, esta diferenciación entre política y policía efectuada por Rancière ha inducido en múltiples lecturas a la creencia de que son polos opuestos y que la policía configura una especie de orden positivo que se ve interrumpido (muy) de vez en cuando por la política, que adquiere en consecuencia un carácter acontecimental, de exterioridad, incluso accidental, que sólo alcanza para criticar lo dado. Nos referimos principalmente a la lectura llevada a cabo por Žižek, quien considera que en la perspectiva rancierana existe una "brecha entre el orden global positivo [...] y las intervenciones políticas que perturban ese orden" (2005: 185). Pero no sólo por él, sino también otros lectores de Rancière incurren en la misma interpretación, por ejemplo, Javier Franzé sostiene:

La política y la policía se diferencian también por su duración o estabilidad. La policía, en tanto momento de reproducción del orden basado en unos esquemas perceptivos-cognitivos, tiende a ser — sobre todo en comparación con la política- lo relativamente 
más consolidado y duradero. La política, en cambio, es sólo el episodio durante el cual se rompe la lógica de la partición policial y se propone una nueva. Como momentánea y contingente que es, la política está condenada a desaparecer como tal, a disolverse en la policía (Franzé, 2012: 65).

Contra esta visión, creemos que la propuesta de Rancière (tal como él mismo ha intentado dejar en claro en varias ocasiones recientes) no pretende marcar tal escisión, sino mostrar la política y la policía como instancias co-constitutivas de un proceso complejo (Reynares, 2011):

no hay de un lado la esfera de las instituciones policiales, y del otro las formas de manifestación puras de la subjetividad igualitaria auténtica [...] La distinción de la política y de la policía opera en una realidad que conserva siempre una parte de indistinción. Es una manera de pensar la mezcla. No hay un mundo político puro y un mundo de la mezcla. Hay una distribución y una redistribución (Rancière, 2004).

Esto permite poner de manifiesto el carácter siempre fallido de todo orden social que se encuentra signado por una distorsión constitutiva, interior a sí mismo, que se pone en evidencia una y otra vez ante la verificación de la lógica igualitaria (política) que viene a reparar "el daño" en la escena pública. Así, la política no es sólo una práctica esporádica que irrumpe sobre una positividad plena, sino una espectralidad permanente que socaba el orden social, es decir, no hay una dicotomía entre momentos policiales puros y momentos políticos puros, ambas lógicas heterogéneas conviven en la cotidianeidad, y el encuentro de ambas (Rancière denomina a eso lo político) no refiere a un momento cronológico determinado y puntual sino a uno conceptual, así el "momento de lo político" tiene una dimensión espectral que es coexistente al orden instituido (Retamozo, 2009).

Este modo de abordar la cuestión nos permite, por un lado, alejarnos de lo que la literatura ha denominado la "diferencia política" entre la política (en Rancière la policía) y lo político (en Rancière la política), al pensar la política como pura administración de lo dado, como 
un ámbito puntual entre otros, como gestión, reproducción y cristalización de un orden; y lo político como acto fundacional, creador, rupturista, instituyente de lo nuevo por otro. Lo que intentamos acentuar con la distorsión constitutiva de todo orden (o en términos de Laclau la dislocación estructural de toda estructura) es que tanto ese momento de primacía de lo policial, de ordenación o gobierno, como ese momento de primacía de lo político, de creación, de transformación, son simultáneos y se afectan mutuamente. Para decirlo mediante un juego de palabras: siempre hay algo de político en lo policial y algo de policial en lo político; esto es, nunca hay, sin más, repetición y reproducción de un orden (volveremos sobre este punto en el último apartado), y todo orden siempre es político; y toda reivindicación igualitaria que emerge no sólo pretende hegemonizarse como nuevo orden policial, sino que inevitablemente excluye a alguien, lo cual configura un nuevo daño. Esto nos lleva a tener que reconocer el conflicto como un elemento central de la institución del orden en la vida comunitaria. Volveremos sobre ese punto más adelante, pero ahora quisiéramos señalar que la intención de la policía es justamente borrar ese componente conflictivo o, lo que es lo mismo, despolitizar su origen, con el fin de naturalizar su modo de distribuir y hacer la cuenta. Pero eso no implica que el conflicto pueda ser eliminado o que el momento de lo político pueda superarse, sino que re-emerge una y otra vez exigiendo un nuevo arreglo de cuentas.

Por otra parte, este modo de concebir la apuesta ranceriana nos permite mirar el escenario argentino de un modo diferente, esto es, ya no pensar sucesos como las jornadas de diciembre de 2001 o cualquier evento de ruptura (institucional-policial) como las únicas instancias en que la política (y el sujeto) se hace presente para luego retornar al orden hegemónico que reabsorbe esa diferencia, Es posible entender que la política está constantemente socavando, cuestionando el orden policial (incluso de manera imperceptible), evidenciando su imposibilidad de estabilizarse de manera definitiva, ${ }^{3}$ lo cual demuestra la politicidad

${ }^{3}$ En este punto la analogía con la teoría de la hegemonía de Ernesto Laclau resulta evidente. No nos detendremos en ello pero lo dejamos planteado para continuar revisando esa relación en futuros trabajos. 
de todo régimen, o bien "verificando la lógica de la igualdad" que destituye toda jerarquía y distribución. Dicho de otro modo, la política y su reparación del daño no es algo que acontece de modo ocasional, de tanto en tanto, sino que es un momento interno de la propia lógica policial que la subvierte a cada instante desde diferentes lugares. Ahora bien, teniendo presente la política como lógica de transformación sin un contenido predeterminado y un lugar prefijado de emergencia, en general la experiencia argentina y latinoamericana de los años recientes nos muestra que el Estado ha cumplido un papel central en esa función (la de reparar el daño y dar lugar a los sin parte), al desplazar el modo de hacer la cuenta, esto nos lleva a nuestro segundo punto.

\section{II}

El segundo aspecto que nos interesa discutir es el lugar del Estado. Éste ha sido sin duda uno de los temas sobre el que la ciencia política ha reflexionado más a lo largo de su historia, teniendo particular atención en el marco latinoamericano. En esa línea, los procesos actuales que se desarrollan en el continente no son la excepción a lo que estamos afirmando. Sin embargo, es precisamente en este punto donde la propuesta de Rancière parece fallar o al menos resultarnos insuficiente. Al hacer un repaso por los principales escritos de su prolífica obra, nos encontramos con que las menciones al Estado por parte del filósofo franco-argelino son más bien marginales cuando no nulas. Su reflexión sobre la democracia o la política no sólo no encuentra en el Estado un referente directo sino que, se podría sugerir, para Rancière aquél se encuentra fuertemente ligado a lo que denomina la "lógica policial", esto es, el modo en que se distribuyen las funciones, los papeles y las tareas en una sociedad.

Lo que estamos marcando sobre Rancière bien podría valer para el posfundacionalismo en general o al menos para una cantidad importantes de los autores que se enmarcan desde esa tradición. Este lenguaje ha sido muy hábil al poner en evidencia y realzar la primacía de lo político como instituyente de todo orden y en mostrar la politicidad de todo acto social más allá de los ámbitos que son canónicamente 
considerados como políticos; dicho de otro modo, ha sido capaz de politizar-deconstruir toda práctica; en última instancia, ha mostrado la precariedad y contingencia de cualquier forma en que se estructura la vida comunitaria.

Decíamos que si bien el posfundacionalismo ha destinado muchas páginas y esfuerzos en destacar todo esto, pareciera haberse excedido en esa empresa y que se olvidó de pensar al Estado o, lo que resulta más problemático, que terminó por reducirlo a la mera reproducción del ordenamiento vigente. Retomando los términos de Rancière, el Estado aparece como mecanismo central en el mantenimiento y la estabilidad del orden policial y la partición que hace de lo sensible. Así, Rancière y buena parte del posfundacionalismo parecieran estar pensando la política en tanto instancia creativa, disruptiva, trasformadora como a distancia del Estado, mientras que en ese complejo entramado institucional sólo habría administración y repetición de lo dado.

Ahora bien, si el Estado tiene un papel eminentemente represivo y ordenador que se opone a la lógica política, emancipadora-igualitaria, dicho punto de partida resulta fuertemente limitante y distorsivo para pensar el lugar del Estado en la América Latina actual, en general, y en Argentina, en particular, sobre todo teniendo presente que ha sido el propio Estado el que ha encabezado y motorizado los procesos de restitución y ampliación de derechos que vienen ejecutándose en los diferentes países de la región y que han irrumpido en el modo en que se venía haciendo la cuenta, dando parte a los sin parte. Por ejemplo, la Asignación Universal por Hijo, ${ }^{4}$ el Plan Conectar Igualdad 5 o la reestatización del sistema previsional, entre muchas otras cuestiones impulsadas

\footnotetext{
${ }^{4}$ Es una asignación familiar establecida por el decreto 1602/09 del Poder Ejecutivo Nacional que se le otorga a las familias desocupadas, que se emplean en el mercado informal o tengan ingresos menores al salario mínimo, vital y móvil por cada hijo menor de 18 años, a cambio del cumplimiento de los controles sanitarios, del plan de vacunación obligatorio y la concurrencia de los menores a un establecimiento educativos público.

${ }^{5}$ Es un programa impulsado por el Poder Ejecutivo Nacional mediante el decreto 459/10 que apunta a reducir la brecha digital y educativa mediante, entre otras actividades, la entrega de notebooks a todos los estudiantes y docentes de establecimientos públicos de educación secundaria, especial y de formación docente para su uso en las aulas y en el hogar particular.
} 
mediante políticas públicas desde el entramado institucional del Estado, sólo desde una mirada excesivamente reduccionista pueden leerse en término de asignación de lugares o bajo una lógica disciplinadora gubernamental de reproducción policial. Evidentemente hay algo que se escapa a ese análisis, algo que excede a la readaptación implacable del orden jerárquico que todo lo asimila y lo vuelve manipulable.

¿Qué hacer entonces? Una posibilidad sería distanciarnos de Rancière entendiendo que su europeísmo no le deja visualizar la politicidad del Estado en otras latitudes. Otra posibilidad, que creemos la más interesante, es precisamente "violentar" el argumento de Rancière y sostener que es en esa ampliación de derechos que viene forzándose desde el Estado que se juega la identidad de los sujetos; que la reivindicación de esas demandas y la asignación de derechos no pueden reducirse a un mecanismo del Estado para clasificar, distribuir y contar a quienes forman parte de la cuenta, y renovar así las formas de exclusión. Al contrario, el reconocimiento de una igualdad antes negada constituye una acción trasformadora de carácter político independientemente de que ésta se satisfaga institucionalmente, ya que subvierte el orden establecido al desarticular los sistemas clasificatorios que distribuyen lugares y otorgan voz, lo que obliga a rehacer la cuenta.

Aquí, la política deja de tener un papel exclusivamente destituyente y comienza a operar en el terreno de lo instituyente e instituido también. Así, el Estado, que para pensar América Latina deviene en central, deja de ocupar el lugar del garante del ordenamiento desigual que implica el orden policial y puede verse como una potencia democratizadora y productora de subjetividades que incluye lo hasta entonces heterogéneo. De este modo, los procesos de democratización de los últimos años parecen enseñarle al propio posfundacionalismo la imposibilidad de esencializar al Estado como reproductor del orden y a los movimientos sociales, las ONG y otras formas de expresión de la sociedad civil como dinamizadores de la lógica política, opuestas al Estado: que éstas asuman una u otra lógica depende del contexto puntual en el que habiten y no hay nada que esencialmente las ubique en una posición fija. La incorporación y participación de movimientos piqueteros y de agrupaciones de lucha por los derechos humanos, históricamente opuestas al Estado, a diversas funciones y áreas de gobierno dan cuenta de ello, 
sin que ese movimiento pueda traducirse a la lógica clientelar (diremos algo más sobre esto en el último apartado).

Lo que estamos señalando aquí puede traducirse sin mayores dificultades en términos de la larga discusión que la literatura ha llevado adelante en torno a la noción de populismo; es decir, la irrupción de la política y los desplazamientos-distorsiones que se producen en el orden policial pueden impulsarse desde el Estado, lo cual da lugar a lo que Ernesto Laclau entiende por lógica populista. Dicho de otro modo, aparece una oferta excesiva por parte del Estado que propone modificaciones que la propia ciudadanía no había logrado impulsar o demandar por sí misma, ${ }^{6}$ es decir, la emergencia de la política como reparadora de un daño no viene impulsada por el colectivo víctima de ese daño o desigualdad (que se constituye en tanto tal a partir de ese acto), sino que proviene desde arriba, desde un Estado que excede sus tareas burocrático-administrativas y comienza configurar nuevos sujetos de derecho al reconocerles una igualdad que ni siquiera se habían atrevido a demandar. ${ }^{7}$ Así, el Estado no sólo puede entenderse como un enemigo o limitante de los derechos, sino que se constituye como momento fundamental de las luchas igualitarias, de corte universalista, en oposición al mantenimiento de los privilegios y las prerrogativas de algunos pocos.

Sin embargo, hay algo más en lo que estamos señalando. Esas políticas que tienen un sentido igualador no son simple ampliación, sino que implican una redefinición de los marcos de sentido, una ruptura sobre la cual es imposible calcular, dimensionar y mucho menos manipular los efectos. En este sentido, los beneficiarios de esas políticas dejan de verse como gobernados que reciben o son alcanzados por las políticas impuestas desde arriba y comienzan a intervenir sobre ellas, a movilizarse a partir de que logran ser interpelados por las acciones estatales-gubernamentales en tanto ciudadanos con derechos susceptibles de participar en la cosa pública con voz propia. La aparición, la ampliación

${ }^{6}$ A esto Eduardo Rinesi lo denomina el componente jacobino del kirchnerismo, de un gobierno que se ubica a la izquierda de la sociedad en la lucha por la igualdad y la ampliación de derechos (Rinesi, 2011).

7 Un buen ejemplo acerca de la lógica de la oferta excesiva es brindado por Alejandro Groppo en su análisis sobre el peronismo: Los dos príncipes: Juan D. Perón y Getulio Vargas (2009). 
y el fortalecimiento de una multiplicidad de agrupaciones militantes cercanas al kirchnerismo durante los últimos años parecen responder a esta lógica. Ciudadanos alejados de las discusiones públicas durante la década de 1990 comienzan a acercarse a la política y a movilizarse como producto de la interpelación que determinadas decisiones gubernamentales produjeron en sus subjetividades. ${ }^{8}$

Es por todo ello que no es posible pensar en ese conjunto de políticas públicas como un mero instrumento de dominación o como tecnología de gobierno para mantener el orden establecido. Podríamos decir entonces que se vuelve posible gobernar emancipando; estas dos palabras que en principios aparecerían disociadas o, más que eso, enfrentadas, ya que la primera responde al conteo de las partes y la segunda a la reivindicación igualitaria de cualquiera con cualquiera en busca de reparar un daño, se vuelven parte de un mismo proceso político en cuanto logramos vislumbrar que el Estado no sólo subordina, restringe y naturaliza, sino que también (y de eso se trata la disputa) ayuda a irrumpir en lo dado y así muestra otros modos en que puede configurarse el mundo.

De este modo, la experiencia latinoamericana de los últimos años, de la que Argentina forma parte, nos permite, parafraseando a Eduardo Rinesi, colocar al Estado "del lado de las cosas malas de la vida y de la historia" (2013: 29), ya que ha generado él mismo la irrupción de la parte de lo que no tenía parte. Por ejemplo, fue desde el Estado que se impulsó la expansión de la lógica de la igualdad y de la universalización de derechos para las trabajadoras domésticas, ${ }^{9}$ y no fueron su organización y su lucha las que consiguieron la ampliación de sus derechos laborales y previsionales. Este tipo de casos, entre muchos otros, dan cuenta del proceso de democratización desarrollado en los últimos tiempos, en el cual el Estado ha asumido un lugar fundamental.

\footnotetext{
8 Para un análisis interesante a partir de entrevistas y declaraciones de los "nuevos militantes", véase el artículo de Gloria Perelló: "Populismo K: un saber hacer con lo imposible" (2007), o el libro de Mauricio Schuttenberg: Las identidades nacional-populares: de la resistencia noventista a los años kirchneristas (2014).

9 Nos referimos a la sanción por parte del Congreso del Régimen de Contrato de Trabajo para el Personal de Casas Particulares, que reconoce a las empleadas domésticas derechos y beneficios amparados por la Ley de Contrato de Trabajo en relación con las jornadas, indemnizaciones, licencias y protección del trabajo de menores.
} 
III

Sin darnos cuenta hemos comenzado a introducir en el final del apartado anterior el tercer punto al que nos interesa hacer referencia aquí: al modo en que el pensamiento rancieriano nos habilita a pensar la democracia. Estamos acostumbrados a entender la democracia en términos de consenso y ligándola a una serie de procesos y mecanismos administrativo-institucionales, o lo que es lo mismo, a pensar la democracia como forma de gobierno, en el cual la confrontación y el conflicto habrían sido superados en favor de grandes acuerdos, apoyados por la totalidad de los integrantes de la comunidad que racionalmente optan por el mejor orden posible. Esta hegemonía de la tradición liberal-representativa para pensar el asunto se apoya en el supuesto de que democracia significa consenso y no conflicto, paz y no enfrentamiento, como también en que las instituciones configuran un conjunto de reglas transparentes, no violentas, que permiten tramitar la vida societal.

La consecuencia de este modo de abordar la cuestión impacta directamente en los casos de análisis que aquí nos interesan. Es común que en los últimos años en Argentina (podríamos extender el diagnóstico a otros países de la región, como Venezuela, Bolivia y Ecuador, cada uno con sus particularidades) se critique al kirchnerismo por confrontar, dividir, crispar, no buscar el consenso. Lo que comparten las diferentes voces (ya sean académicas, partidarias, periodísticas, etcétera) que sostienen esas críticas es la visión de la democracia que acabamos de enunciar en el párrafo anterior, es decir, sus incomodidades no apuntan a cuestionar la democracia, sino que intentan señalar que esos gobiernos, en tanto exacerbadores del conflicto, estarían atentando contra el espíritu mismo de la democracia (el consenso) y sus formas institucionalizadas. Dicho de otro modo, el conflicto no sería un aspecto constitutivo de la democracia sino un ataque contra los acuerdos entre las partes integrantes de la sociedad.

Desde la mirada de Rancière, este tipo de posturas que esencializan la democracia a su componente consensual se leerían como la forma que en la actualidad adopta el odio a la democracia que ha atravesado el pensamiento político occidental (siempre preocupado por el mantenimiento del orden y la eliminación del conflicto) desde sus comienzos 
en la Grecia clásica. La democracia ha suscitado ese odio al constituirse como un escándalo para el pensamiento en tanto que representa la igualdad de cualquiera con cualquiera; la pérdida de privilegios y distinciones; la posibilidad de que cualquier sujeto sin ningún tipo de título o atributo que lo distinga reclame y se arrogue la capacidad de participar en la esfera pública como un par, de hacerse contar y tomar la palabra en el proceso de toma de decisiones colectivamente vinculantes (Rancière, 2007a). La democracia implica una apertura a lo azaroso, un desafío a quienes creen tener las virtudes y el poder para decidir por sobre el resto, un ejercicio desmesurado que muestra la ausencia de fundamento de todo ordenamiento. Es por ello que se pretende limitar el ejercicio democrático, reintroduciendo nuevos criterios de ordenamiento jerárquico o reduciéndolo a su mínima expresión (las elecciones periódicas), controlando la "vida democrática", orientándola a los fines de la vida privada para evitar su exceso (Rancière, 2007a). Así, para Rancière, quienes hoy se autonominan como defensores de la democracia y de las instituciones y oponerse a las confrontaciones son en realidad sus principales enemigos.

Esto que venimos marcando de la mano de Rancière permite vislumbrar un modo distinto de aproximarnos a la democracia. A partir de lo dicho, la democracia en tanto modo de reactualización de la lógica igualitaria en las relaciones sociales no es otra cosa que la manifestación de la política en los términos que hemos señalado antes. Esa reinscripción igualitaria no puede efectuarse libre de tensiones y de conflictos, dado que implica la destitución de un orden policial vigente que desigualaba, lo cual produciría una pugna, una disputa, un enfrentamiento por la reconfiguración del orden, por el modo de hacer la cuenta. En síntesis, la violencia simbólica es inherente al proyecto democrático, el conflicto es constitutivo de su accionar:

democracia es el nombre de una interrupción singular de ese orden de distribución de los cuerpos en comunidad que se ha propuesto conceptualizar con el empleo de la noción ampliada de policía. Es el nombre de lo que viene a interrumpir el buen funcionamiento de ese orden a través de un dispositivo singular de subjetivación (Rancière, 2010a: 126). 
El tan mentado consenso, visto así, pretende despolitizar la democracia al negar la lucha entre órdenes inconmensurables e irreductibles a la armonía, presuponiendo que todos los actores tienen palabra autorizada, se encuentran en condiciones de isonomía y pueden participar en el debate público (vimos en el primer apartado que la discusión es anterior, en tanto que la discusión misma es por quién se considera capacitado y por los presupuestos implícitos en estas miradas: que la comunicación es posible, que podemos comprender lo que los otros dicen y elegir una opción racionalmente óptima para todos, aunque no se verifican en la realidad). Contra ello, la democracia podría pensarse como un proceso de lucha por la igualdad real y el acceso a esa palabra pública, en tanto que el orden existente no nos reconoce como iguales ni es neutral. Así, la dinámica democrática en tanto actualización de la lógica igualitaria implica "una fractura primera por la que se introducen en la comunidad de seres parlantes aquellos que no estaban incluidos" (Rancière, 2007a: 67).

Por otra parte, lo dicho nos obliga a distanciarnos de pensar la democracia como una forma jurídico-política, restringida al Estado, o como un ordenamiento constitucional, para acercarnos a pensar la democracia en términos de un movimiento permanente, como una desmesura ingobernable que cuestiona todo límite y fundamento en que se basa toda estructura y como un proceso de lucha por el ensanchamiento de la esfera pública. Esto puede traducirse en un reconocimiento entre iguales de aquellos sujetos dañados a los que el sistema policial repelía hacia su vida privada como seres inferiores. En este sentido, la ley de identidad de género sancionada en 2012 que permite, entre otras cosas, a todas las personas trans elegir el nombre y el sexo que llevarán en su documento nacional de identidad es un caso evidente de la lucha por tal ampliación democrática que estamos intentando afirmar. En la misma línea, el reconocimiento del carácter público de espacios y las relaciones antes subsumidas a la discreción del poder de la riqueza o al ámbito privado, lo cual extiende la igualdad a cada vez más esferas, puede verse en la jubilación de las amas de casa, que reconoce el trabajo doméstico antes invisibilizado o en la institucionalización del voto joven, que habilita a elegir a sus representantes en los procesos eleccionarios nacionales a los jóvenes de 16 y 17 años 
que voluntariamente así lo deseen. Esto implica la puesta en marcha de una igualdad que no se reconocía previamente. ${ }^{10}$ Así, se permite la emergencia de nuevos sujetos que hacen propio algo que habían naturalizado como ajeno, y se abren así nuevas luchas. En este sentido, el kirchnerismo puede ser leído como un proceso democrático (en el sentido que venimos construyendo aquí) que impulsa el conflicto al desnaturalizar lo dado y que politiza lugares anteriormente fijados.

En resumen, más allá de los procedimientos y mecanismos de selección de gobernantes, para Rancière la democracia es el gobierno de aquellos que no tienen ningún título más que su reivindicación como iguales. Es precisamente ese aspecto el que escandaliza a los sectores que se arrogan algún otro atributo (riqueza, conocimiento, poder, etcétera) a partir del cual pretenden gobernar, ya que al ver la igualdad puesta en marcha en tanto manifestación de la política, buscan limitarla ya sea a través del gobierno de la técnica, del consensualismo o del mero formalismo. En este sentido, la democracia liberal impuesta en nuestras latitudes no es otra cosa que un intento de aquellos que odian la democracia, por domesticarla y contenerla según sus pretensiones. Así, podríamos pensar las experiencias que nuestros países están atravesando como la encarnadura misma del desacuerdo sobre la democracia, que convocan a hablar a aquella parte que no tenía voz, ni se le reconocía capacidad y que en nombre del daño recibido reclama ser tenida en cuenta como el representante de la totalidad.

\footnotetext{
$10 \mathrm{Al}$ respecto, es interesante remarcar la forma que asumió la discusión por dicha modificación. Los sectores que apoyaban la ampliación del voto para los jóvenes de 16 y 17 años sostenían el reconocimiento de la igualdad de ese colectivo con el resto de los votantes, lo cual otorgaba un derecho donde antes había una negación y repercutía en una ampliación en las formas de participación y en una legitimación de la voz de los beneficiarios. Los sectores que se oponían a tal medida manifestaban la incapacidad de los jóvenes de 16 y 17 años para participar de las elecciones, la facilidad con que podían ser manipulados e influenciados y su falta de conocimiento, es decir, se los situaba en un lugar de inferioridad. Lo que la estructura de este debate pone en evidencia se vincula con los dos modos que venimos señalando que están en disputa al momento de pensar la democracia: una preocupada por ampliar las esferas de igualdad cuestionando los límites establecidos y naturalizados, y otra basada en la justificación de las diferencias sostenidas con títulos como el conocimiento, la edad, etcétera.
} 
Pensar la democracia como algo distinto del liberalismo es un ejercicio que buena parte de la ciencia política y las ciencias sociales en general han dejado de lado, fruto de la hegemonía liberal después del fin de la Guerra Fría. En este aspecto, como señala Mouffe (en consonancia con Rancière) una democracia se ve favorecida ante la exacerbación del conflicto y los antagonismos entre visiones diferentes que se traducen en una esfera pública vibrante, lo que no es otra cosa que una comunidad en litigio por la verificación de la igualdad (Mouffe, 1999).

IV

Finalmente, quisiéramos dedicar estas últimas páginas a realizar una serie de reflexiones en torno a la noción de sujeto y su relevancia a la luz de la experiencia argentina de los últimos años. Rancière nos propone pensar al sujeto como un entremedio, como un in between entre dos identidades que pugnan por estabilizarse y darle sentido a las acciones de los individuos. Es justamente en ese intervalo que, según el autor franco-argelino, puede emerger el sujeto político. Así, éste sería la consecuencia de dos procesos o momentos que se producen simultáneamente. Por un lado, la desidentificación frente al lugar asignado, la desclasificación en relación con lo esperable desde la mirada del orden imperante, correrse o no asumir los papeles impuestos por lo dado (o lo que es igual, por la lógica policial); por otro lado, el comienzo de un nuevo polo de (re)identificación, que no se puede asumir como tal porque todavía no existe (Rancière, 2000), es decir, significa asumir un nombre impropio, polémico, que no tiene lugar dentro del orden policial y que en última instancia resulta imposible de constituir a menos que se pague el precio de ser sometido nuevamente a un orden policial (diferente, pero tan policial como el anterior) que le asigne un lugar en la cuenta, que lo cristalice en una identidad nueva. ${ }^{11}$ De este modo, el sujeto no es ninguno de los dos

11 Para graficar este punto ha sido recurrentemente utilizado el ejemplo brindado por el propio Rancière (2000) sobre la experiencia de Auguste Blanqui. Este revolucionario francés del siglo xix, en ocasión de un juicio en su contra, ante la pregunta del 
elementos que acabamos de marcar, no es ni pura desidentificación ni pura identidad nueva (imposible), es precisamente en el intervalo, en esa mitad de camino entre ambos que emerge. Es ese doble movimiento el que vuelve visible lo hasta entonces invisible, que hace aparecer el daño hasta entonces ignorado que el sistema le efectuaba a la parte excluida. ${ }^{12}$

De esta manera, Rancière sostiene que "la lógica de la subjetivación es una heterología, una lógica del otro", ya que el sujeto no afirma simplemente una identidad, lo que abre un espacio novedoso, sino que esa constitución del sujeto siempre está en relación con un otro, incluso ante el rechazo de este último; se instituye así una comunidad basada en el litigio. El sujeto siempre se dirige a otro, porque siempre implica una identificación imposible, alude a una posición cuya manifestación rompe la distribución jerárquica vigente (Rancière, 2000).

Ahora bien, enunciado como acabamos de hacerlo, nos encontramos con un escenario bastante similar al que trazamos en el primer apartado de este trabajo sobre la relación entre política y policía. Nos referimos a que, tal como muchos han interpretado, para Rancière el sujeto asumiría la forma de una aparición esporádica, fugaz, puntual,

fiscal sobre su profesión decide responder "proletario". La réplica policial fue: "esa no es una profesión". Ante lo que Blanqui devolvió: "es la profesión de la mayoría de nuestro pueblo a la que se la ha privado de derechos políticos" (Rancière, 2000: 148). Ese pequeño gesto de Blanqui, que podría pasar desapercibido o ser leído como una simple fanfarronada, muestra para Rancière la aparición del sujeto político de manera muy evidente. Por un lado, se sustrae del lugar asignado por el orden policial, de las respuestas esperables, de los nombres que le eran propios. Por otro, comienza a configurar una identidad imposible, ya que Blanqui en sentido estricto no era un proletario y el proletariado como tal es una identidad que no existe en sentido sociológico, es el nombre (metafórico podríamos decir, es el significante vacío en términos de Laclau) de todos aquéllos a los que se les niega su parte, su identidad, sus derechos (Rancière, 2000).

12 Según Franzé, ese modo de visibilizar el daño puede asumir dos formas: la primera es una exteriorización de un "sí mismo" invisibilizado, víctima de un daño; la segunda responde a una interiorización de otro invisibilizado que luego implica una exteriorización de ello; sería la manifestación de un daño sobre otro que considero un daño sobre mí mismo y me hace rechazar la cuenta en la que estaba incluido. La expresión típica del primer camino de subjetivación sería: “iuna francesa es un francés?", mientras que la de la segunda sería: "todos somos judíos alemanes" (2010: 65). 
simultánea al momento de irrupción de la política y su lógica igualitaria en el escenario público. Ese episodio dislocador dejaría espacio para que rápidamente una reconfiguración policial se produjera y conformara un nuevo orden y distribución de lugares y partes, en donde la política se habría desvanecido y con ella el sujeto que la encarnaba. Así, las interpretaciones acontecidas de Rancière entienden que la política y el sujeto representan un evento o momento acotado en el tiempo, durante el cual la lógica de la partición jerárquica policial se interrumpe para luego dar lugar a la consolidación y estabilidad de un nuevo régimen policial que absorbe al sujeto que reclamaba (asignándole un lugar) y disuelve a la política en la pura administración y gestión.

Este tipo de lecturas de Rancière son susceptibles de ser criticadas bajo la misma premisa que Aletta Norval empleaba para mostrar las limitaciones de la concepción de sujeto de Laclau. Esta autora señala que el intelectual argentino desarrolla una "concepción heroica del sujeto" al sostener que existen, por un lado, "posiciones de sujeto", es decir, identidades sedimentadas al interior del discurso hegemónico vigente, como resultado de la participación en la vida social cotidiana; por otro lado, el "sujeto" en sentido estricto como un agente político autónomo. Con esta distinción, lo que Norval intenta poner en evidencia es que, desde la perspectiva de Laclau, el sujeto sólo aparecería en esas excepcionales oportunidades en las que nuevos discursos logran instituirse y configurar identidades populares a partir del antagonismo (Norval, 2006). La consecuencia de ello es que habría una suerte de despolitización de la vida cotidiana, la cual se convierte en la mera reproducción de lo dado y frente a la cual sólo queda esperar la llegada del momento de irrupción y redención de lo político que habilitaría la aparición del sujeto. No habría a nivel micro y en la cotidianeidad la posibilidad de un sujeto político y de la acción desnaturalizadora de la política que queda reservada a los grandes acontecimientos.

El énfasis en una ruptura radical con los arreglos institucionales existentes deja lo político fuera de la política de todos los días en los órdenes políticos altamente institucionalizados en que los sujetos parecen ser sólo seguidores ciegos de racionalidades-administrativas limitadas. Pero si toda estructura está inevitablemente abierta y configurada de modo contingente, la política no puede ser más que parte integrante 
de las instituciones tanto en tiempos de crisis como en épocas de estabilidad. Esto no implica desconocer que la política juega un papel más autónomo cuando los sistemas institucionales están en crisis y la acción humana puede liberarse de las restricciones institucionales. Sin embargo, es importante insistir en que el énfasis con el que la constitución de subjetividades políticas exige una ruptura radical con los arreglos institucionales existentes desdibuja la importancia de la política cotidiana para hacer posible no sólo el mantenimiento, sino también la reactivación y redefinición del momento inicial de identificación política (Norval, 2006).

Retomemos ahora nuestro argumento sobre Rancière. Decíamos que nuestro autor ha sido leído mayoritariamente bajo la lógica heroica del sujeto que emerge en un momento esporádico para mostrar el daño efectuado a la presuposición de la igualdad y después someterse al orden policial siguiendo sus determinaciones de modo acrítico, reproduciendo lo establecido. Contra ese conjunto de interpretaciones, siguiendo la misma línea argumental sostenida en el primer apartado, creemos que Rancière y su propuesta del sujeto como intervalo no nos conduce necesariamente a entenderlo de ese modo, sino que el sujeto irrumpe mucho más frecuentemente y de modo mucho más sutil de lo que estas miradas estarían dispuestas a asumir. Creemos que es por ello que podemos entender a nuestro autor como un intelectual del gesto más que del acontecimiento. Nos apoyamos para ello en los múltiples ejemplos que Rancière desarrolla a lo largo de su obra, los cuales nunca están planteados como grandes sucesos o trascendentes actos de individuos de renombre o recordados por la historia tradicional, sino más bien como pequeños gestos y momentos que pueden juzgarse como secundarios o parecer no tener relevancia, pero que encierran una ruptura o subversión ante el modo en que se cuentan las partes. ${ }^{13}$ El uso de esos casos mínimos

13 Estamos pensando en los ejemplos ya antes citados como el de Blanqui, pero también en el caso de las mujeres francesas del siglo xix que en nombre de la igualdad reclaman la posibilidad de ser candidatas a cargos políticos; en la disputa entre plebeyos y patricios en el Aventino y su reconocimiento como seres con lenguaje; en los relatos de La noche de los proletarios (Rancière, 2010b) sobre las actividades realizadas por los trabajadores franceses durante las noches para recuperar las horas que el trabajo les impedía aprovechar; en el reclamo de los trabajadores franceses del siglo XIX 
dan cuenta de que la aparición del sujeto, de la que nos habla Rancière, no requiere de una revolución (en el sentido tradicional del término) o de transformaciones radicales que arrastren consigo la desigualdad policial, sino que son pequeños desplazamientos que ocurren, a veces, de modo imperceptible incluso en el interior del mismo orden policial.

Lo que estamos señalando nos lleva nuevamente a resaltar que política y policía no pueden ser pensados como dos entidades positivas puras de carácter dicotómico, sino como dos lógicas que se implican mutuamente, y que es en esa tensión permanente que el sujeto político (y la política) emerge una y otra vez desplazando los sentidos y papeles fijados. A partir de ello es que nunca existe una simple repetición o subordinación ante la distribución impuesta, sino la iteración que produce corrimientos sutiles (insistimos, a menudo imperceptibles) que afectan y modifican el orden establecido. Evidentemente, ante "crisis orgánicas" la aparición del sujeto (y de la política) se hace más palpable, pero eso no implica que en momentos de estabilidad institucional-policial no se produzca ese entremedio entre la desidentificación y la reinscripción en una nueva identidad.

Ahora bien, existe un punto más a destacar. Retomando lo esbozado en el segundo apartado del presente trabajo, el Estado no puede pensarse como reducido a un mero reproductor del orden sino que es también, la experiencia latinoamericana lo ratifica, un espacio desde el cual es posible hacer emerger la política y demandar en nombre del daño que se les hace a los excluidos, así como configurador de sujetos que reclaman ser tenidos en cuenta. Dicho de otro modo, los nuevos derechos adquiridos en los últimos años por los ciudadanos de los diferentes países de la región no implican simplemente la distribución de lugares y la asignación de nuevos papeles por parte de un orden policial, son también la reparación de un daño que los sujetos no asumen de manera acrítica y manipulable, sino que aceptan y reclaman más. Esto nos permite corrernos de las miradas que suponen una lógica clientelar de intercambio entre demandas y políticas públicas, que conduce a la

que demandaban ser tratados como iguales a sus empleadores; en la desidentificación de algunos intelectuales franceses frente al bombardeo del gobierno francés en Argelia; entre muchos otros casos brindados por el propio autor. 
subordinación ante el orden establecido y su reproducción, y comenzar a pensar en términos de una heteroautonomía del sujeto. Esto no sólo significa que el Estado, con sus decisiones gubernamentales, disciplina y reproduce, sino que los ciudadanos receptan esas políticas y desplazan, mediante esos pequeños gestos, exigiendo ir más allá, y allí puede vislumbrarse la emergencia del sujeto, que imposibilita el completo cierre de todo orden policial-institucional.

Tomemos un ejemplo breve para cerrar. La institución del matrimonio igualitario ${ }^{14}$ en Argentina difícilmente puede leerse como una metamorfosis del orden policial-estatal por continuar con la distribución sensible vigente, más bien, puede entenderse como su opuesto, como una ruptura política impulsada desde el Estado, que implica la obtención de derechos y la subsiguiente visibilización de lo hasta entonces borrado por la partición sensible, pero, además de ello, esta aparición de lo político no implica la posterior desaparición del sujeto, sino un permanente desplazamiento, ampliación y habilitación de las prácticas e instituciones establecidas. Así, es posible entender, por ejemplo, que los mismos colectivos comprometidos con el matrimonio igualitario, que ahora se hacen contar como parte en el orden comunitario, no se han transformado al seguidismo del gobierno nacional, sino que desde sus lugares hacen propia la posibilidad de casarse, afectan otras prácticas, exigen ir más allá, impulsan luchas y demandas contiguas como la despenalización del aborto, las leyes de educación sexual, las leyes de fertilización asistida, entre otras.

\section{ReFLEXIONES FinALES}

En estas últimas líneas recapitularemos algunas ideas centrales planteadas a lo largo del trabajo. El propósito fue tomar las categorías de la propuesta ranceriana, tensionarlas y ponerlas en juego para pensar la experiencia kirchnerista de los últimos años en Argentina. El primer punto presentó la lógica policial y política como procesos co-constitutivos que

\footnotetext{
${ }^{14}$ Nos referimos a la ley 26.618 que habilita el matrimonio a personas del mismo sexo y permite la adopción a parejas homosexuales.
} 
están en permanente interacción y contaminación. Esto nos habilita para pensar, desde las ideas de Rancière, en términos políticos todo episodio coyuntural y no limitarnos a esperar la llegada del acontecimiento como irrupción momentánea de la política. Al reconocer la distorsión constitutiva de la realidad, evitamos caer en la despolitización a la que nos tienta la lógica policial, admitiendo la institución política de toda práctica. El segundo aspecto mostró la centralidad del Estado en los procesos de ampliación de la igualdad ocurrida en los últimos años. Esto nos obliga a dejar de pensar el Estado como el agente policial por excelencia (que lo es), sino (también) entenderlo como un actor que con sus complejidades, contradicciones y entramados puede poner en marcha el presupuesto de la igualdad. El tercer apartado se destinó a recuperar la noción de Rancière sobre la democracia como el modo mismo en que se desenvuelve la lógica igualitaria, más allá de los procedimientos y las dinámicas institucionales, reconociendo que tal ejercicio implica un componente conflictivo inerradicable que otras tradiciones de pensamiento se niegan a admitir y buscan ocultar. Finalmente, se sostuvo que la propuesta del sujeto como intervalo entre identidades resulta productiva, en la medida en que nos alejamos de su lógica heroica y lo pensamos en términos cotidianos, como un sujeto que se desidentifica y comienza a construir nuevos polos de identidad, mediante pequeños gestos o desplazamientos de la prácticas con las que cuenta día a día en la coyuntura, sin que se produzcan necesariamente grandes sucesos para que su accionar subversivo pueda producirse.

Estos cuatro puntos nos ayudan a pensar al kirchnerismo de un modo diferente a como los análisis canónicos provenientes de la ciencia política tradicional lo viene haciendo; eso es lo que hemos intentado mediante los casos citados y los ejemplos utilizados. Así, al tensionar las categorías del propio Rancière podemos pensar al kirchnerismo como un proceso político-democrático, en el sentido de que tensiona el orden policial establecido, impulsa la lógica de la igualdad desde el Estado y abre desde arriba la necesidad de rehacer la cuenta para ampliar derechos y habilitar la emergencia de nuevas subjetividades. 
FuENTES CONSULTADAS

Franzé, J. (2010), "La política como proceso de subjetivación: un estudio de caso sobre los inmigrantes latinoamericanos en Madrid", en Rosa de la Fuente Fernández (ed.), Migración y política: latinoamericanos en la comunidad de Madrid, Madrid: Trama, pp. 61-82.

Mouffe, C. (1999), El retorno de lo político, Barcelona: Paidós.

Norval, A. (2006), "Democratic Identification: A Wittgensteinian Approach", en Political Theory, vol. 34, núm. 2, abril, pp. 229255.

Rancière, J. (2000), "Política, identificación, subjetivación", en Benjamín Arditti (ed.), El reverso de la diferencia. Identidad y política, Caracas: Nueva Sociedad. pp. 145-152.

RANCIÈre, J. (2004), El uso de las distinciones. Disponible en [http://www. ddooss.org/articulos/otros/Jacques_Ranciere.htm].

RAncière, J. (2007a), El odio a la democracia, Buenos Aires: Amorrortu.

RANCIĖRE, J. (2007b), El maestro ignorante, Buenos Aires: Libros del Zorzal.

Rancière, J. (2010a), El desacuerdo. Política y filosofía, Buenos Aires: Nueva Visión.

Rancière, J. (2010b), La noche de los proletarios, Buenos Aires: Tinta Limón.

RANCIĖRE, J. (s.f.), En los bordes de lo político [edición digital], traducción de Alejandro Madrid Zan y José Grossi, Santiago de Chile: Escuela de Filosofía-Universidad de Arte y Ciencias Sociales (Arcis).

Retamozo, M. (2009), "Lo político y la política: los sujetos políticos, conformación y disputa por el orden social", en Revista Mexicana de Ciencias Políticas y Sociales, núm. 206, México: Universidad Nacional Autónoma de México (unAm), pp. 69-91.

Reynares, J. M. (2011), “Ontología de la distorsión”, en Emmanuel Biset y Roque Farrán (comps.), Ontologías políticas, Buenos Aires: Imago Mundi, pp. 101-122.

Rinesi, E. (2011), "Notas para una caracterización del kirchnerismo", en Debates y Combates, año 1, núm. 1, noviembre, Buenos Aires: Fondo de Cultura Económica (FCE), pp. 141-172. 
RINESI, E. (2013), "De la democracia a la democratización: notas para una agenda de discusiones filosófico-política sobre los cambios en la Argentina actual. A tres décadas de 1983", en Debates y Combates, año 3, núm. 5, julio-agosto, Buenos Aires: FCE, pp. $19-42$.

ŽıžEK, S. (2005), El espinoso sujeto. El centro ausente de la ontología política, Buenos Aires: Paidós.

Fecha de recepción: 15 de diciembre de 2014 Fecha de aprobación: 11 de mayo de 2016 